\title{
AVERAGE TREE SOLUTION AND SUBCORE FOR ACYCLIC GRAPH GAMES
}

\author{
Dolf Talman \\ Tilburg University
}

\author{
Yoshitsugu Yamamoto \\ University of Tsukuba
}

(Received December 28, 2007)

\begin{abstract}
In this paper we consider cooperative transferable utility games with limited communication structure, called graph games. Agents are able to cooperate only if they can communicate directly or indirectly with each other. For the class of acyclic graph games the average tree solution has recently been proposed. It was proven that the average tree solution is a core element if the game exhibits super-additivity. We show that the condition of super-additivity can be relaxed to a weaker condition, which admits for a natural interpretation. Moreover, we introduce the concept of subcore, which is a subset of the core, always contains the average tree solution, and therefore is a non-empty refinement of the core.
\end{abstract}

Keywords: Game theory, graph theory, limited communication structure, acyclic graph, super-additivity, core

\section{Introduction}

In many economic situations agents are able to obtain more profits or save costs by cooperation. For example, by sharing certain facilities (catering, security, communication systems, transportation) firms may obtain higher total payoffs. The total maximum additional payoff a subgroup of agents, also called a coalition, can obtain from cooperation is called its worth. If the worth of a coalition can be freely distributed amongst its members (transferable utility), the problem becomes how much payoff every agent (player) should get. A classical set-valued solution is the core, see Gillies [3], being the set of payoff distributions (payoff vectors) at which the worth of the whole set of players (the grand coalition) is distributed amongst the players (efficiency) and no coalition receives less than its worth (non-domination). If a payoff vector is not an element of the core, some coalitions can do better than that by their own. The most well-known single-valued solution is the Shapley value, see Shapley [6]. At the Shapley value every agent receives the (weighted) average of all his marginal contributions to any coalition that he is a member of. The Shapley value, however, may not be an element of the core.

In this research we study cooperative games with limited communication structure represented by an undirected graph. These so-called graph games were introduced by Myerson [5]. A group of players is only able to cooperate if they can communicate directly or indirectly with each other. The best-known single-valued solution for such games is the Myerson value, being characterized by efficiency and fairness. In Borm et al. [1] the so-called positional value is proposed. This value is characterized by efficiency and balanced total threats, see Slikker [7]. In Herings et al. [4] the average tree solution is introduced for the class of acyclic graph games. The average tree solution is characterized by efficiency and component fairness. Component fairness means that deleting a link between two players yields for both 
resulting components the same average loss in payoff, whereas fairness says that deleting a link gives the same loss in payoff for both end points of the link. The average tree solution is the average of some specific marginal contribution vectors. For super-additive acyclic graph games all these vectors lie in the core and therefore also the average tree solution is an element of the core. For this class of games the Myerson value and the position value may not be elements of the core.

In this paper we give a condition for the characteristic function that is weaker than super-additivity to make the average tree solution be an element of the core. Moreover, we refine the core to a smaller subset, called the subcore, and show that for the class of acyclic graph games satisfying this weaker condition for the characteristic function the average tree solution is always an element of the subcore and therefore that the subcore is a non-empty refinement of the core. Section 2 introduces the class of graph games. Section 3 relates the average tree solution to the core and Section 4 introduces the subcore.

\section{Preliminaries}

We consider cooperative games with limited communication structure, called graph games, as has been introduced by Myerson [5]. A graph game is represented by a triple $(N, v, E)$, where $N$ is a finite set of $n$ players, $v$ is a characteristic function that assigns the worth to coalitions, and $E \subseteq\{\{i, j\} \mid i, j \in N, i \neq j\}$ is a collection of communication links between players.

The pair $(N, E)$ is called an (undirected) graph with $N$ the set of nodes, being the players of the game, and with $E$ the collection of edges (links) between the nodes. In case $E=\{\{i, j\} \mid i, j \in N, i \neq j\}$ the game $(N, v, E)$ is said to have full communication structure and is simply denoted by $(N, v)$.

A sequence of nodes $\left(i_{1}, \ldots, i_{k+1}\right)$ is called a cycle in a graph $(N, E)$ if (i) $k \geq 3$, (ii) all nodes $i_{1}, \ldots, i_{k}$ are different elements of $N$, (iii) $i_{k+1}=i_{1}$, and (iv) $\left\{i_{h}, i_{h+1}\right\} \in E$ for $h=1, \ldots, k$. A graph is said to be acyclic when it does not contain any cycle. A set of nodes $K \subset N$ is said to be connected in the graph $(N, E)$, if for any two distinct nodes $i, j \in K$ there exists a sequence $\left(i_{1}, \ldots, i_{k}\right)$ of nodes in $K$ satisfying $i_{1}=i, i_{k}=j$ and $\left\{i_{h}, i_{h+1}\right\} \in E$ for all $h=1, \ldots, k-1$. A subset $K$ of $N$ is called a component of the graph $(N, E)$ if $K$ is connected in $(N, E)$ and for any $j \in N \backslash K$ the set $K \cup\{j\}$ is not connected in $(N, E)$. For the graph $(N, E)$ and a subset $H \subset N$, the set $E(H)$ is given by $E(H)=\{e \in E \mid e \subset H\}$. The collection of connected subsets of $K$ in the subgraph $(K, E(K))$ of a graph $(N, E)$ is denoted by $C^{E}(K)$ and the collection of components of $(K, E(K))$ is denoted by $C_{m}^{E}(K)$.

In this paper it is assumed without loss of generality that in a graph game $(N, v, E)$ the set $N$ is always connected in the graph $(N, E)$, i.e., $N \in C^{E}(N)$. Due to the limited communication, members of a coalition $S \in 2^{N}$ are able to cooperate only if all members of $S$ can communicate directly or indirectly with each other, i.e., $S \in C^{E}(N)$. For $S \in C^{E}(N)$, the worth $v(S)$ is the maximum amount of payoff a coalition $S$ can obtain for its members. Concerning the characteristic function $v$, the graph game is said to exhibit super-additivity if

$$
v(S \cup T) \geq v(S)+v(T)
$$

for all $S, T \in C^{E}(N)$ satisfying $S \cap T=\emptyset$ and $S \cup T \in C^{E}(N)$.

A payoff vector $x$ is an $n$-dimensional vector giving payoff $x_{i}$ to player $i \in N$. For simplicity we denote $x(S)=\sum_{i \in S} x_{i}$ for $S \in 2^{N}$. For a graph game $(N, v, E)$ a payoff vector $x$ is said to be efficient if $x(N)=v(N)$. The core, denoted by $C(N, v, E)$, of a graph game $(N, v, E)$ is the set of efficient payoff vectors that are not dominated by any connected 
coalition,

$$
C(N, v, E)=\left\{x \in R^{n} \mid x(N)=v(N) \text { and } x(S) \geq v(S) \text { for all } S \in C^{E}(N)\right\} .
$$

The core of a game $(N, v)$ with full communication is denoted by $C(N, v)$, i.e.,

$$
C(N, v)=\left\{x \in R^{n} \mid x(N)=v(N) \text { and } x(S) \geq v(S) \text { for all } S \in 2^{N}\right\} .
$$

Notice that the core $C(N, v, E)$ of a graph game $(N, v, E)$ equals the core $C\left(N, v^{E}\right)$ of the so-called restricted game $\left(N, v^{E}\right)$ with full communication, defined by Myerson [5] as

$$
v^{E}(S)=\sum_{T \in C_{m}^{E}(S)} v(T) \quad \text { for } S \in 2^{N}
$$

For a permutation $\pi=(\pi(1), \ldots, \pi(n))$ of $N$, the marginal contribution vector $m^{\pi}(v) \in$ $R^{n}$ assigns to every player $i$ payoff $m_{i}^{\pi}(v)=v\left(\pi^{i} \cup\{i\}\right)-v\left(\pi^{i}\right)$, where $\pi^{i}=\{j \in N \mid$ $\pi(j)<\pi(i)\}$ is the set of players preceding $i$ in the permutation $\pi$. The most well-known single-valued solution for the class of cooperative games with full communication is the Shapley value, which assigns to game $(N, v)$ the average $\psi(N, v)$ of all its marginal vectors. Although the Shapley value is an efficient solution, it may, however, not be an element of the core, even if the core is non-empty.

The most well-known single valued solution for the class of graph games is the Myerson value $\mu(N, v, E)$, due to Myerson [5]. The Myerson value assigns to a graph game $(N, v, E)$ the Shapley value $\psi$ of the corresponding restricted game, i.e., $\mu(N, v, E)=\psi\left(N, v^{E}\right)$. The Myerson value of a graph game $(N, v, E)$ is not always an element of the core, even not if the graph $(N, E)$ is acyclic and the game itself is super-additive. From Demange [2] it is known that the core of a super-additive acyclic graph game is non-empty because it contains several specific marginal contribution vectors, but not always all.

\section{The Average Tree Solution and the Core}

For the class of graph games the average tree solution was introduced by Herings et al. [4]. To describe the average tree solution we first give some definitions concerning directed graphs. A graph $(N, D)$ is directed if $D \subseteq N \times N$, i.e., $D$ is a set of ordered pairs of nodes. An ordered pair of nodes is called an arc. For $D \subseteq N \times N$ let $\bar{D}=\{\{i, j\} \mid(i, j) \in D\}$. A directed graph $(N, D)$ is said to be acyclic* if the undirected graph $(N, \bar{D})$ induced by $D$ is acyclic and $(N, D)$ is said to be connected if $(N, \bar{D})$ is connected.

Definition 3.1. An acyclic connected directed graph $(N, D)$ is said to be an arborescence if each node has at most one arc entering the node.

Clearly, an arborescence has exactly one node that no arc enters, which is called the root, and there is a unique directed path from the root to each node. For a given arborescence $(N, D)$, for each node $i \in N$ we define its sets of successors and descendants as

$$
\operatorname{suc}(i)=\{j \in N \mid(i, j) \in D\}
$$

and

$$
\operatorname{des}(i)=\{j \in N \mid j=i \text { or there is a directed path from } i \text { to } j\},
$$

*This usage of acyclic is not common. 
respectively. We also define inductively the height $\tau(i)$ of node $i \in N$ as follows:

$$
\tau(i)= \begin{cases}0 & \text { if } \operatorname{suc}(i)=\emptyset, \\ 1+\max _{j \in \operatorname{Suc}(i)} \tau(j) & \text { otherwise. }\end{cases}
$$

For a given acyclic graph game $(N, v, E)$ the tree solution, denoted by $x^{r}$, with respect to node $r \in N$, is defined as follows.

Step 1: Make an arborescence $D^{r}$ with node $r$ as root. Set $t:=0$.

Step 2: If there is no node $i \in N$ with $\tau(i)=t$, then terminate.

Step 3: For each node $i \in N$ with $\tau(i)=t$ set

$$
x_{i}^{r}:=v(\operatorname{des}(i))-\sum_{j \in \operatorname{des}(i) \backslash\{i\}} x_{j}^{r} .
$$

Step 4: Set $t:=t+1$ an go to Step 2 .

Since the graph $(N, E)$ is acyclic, the arborescence $\left(N, D^{r}\right)$ with root $r$ is uniquely determined. More precisely, if $\left(i_{1}, \ldots, i_{k}\right)$ is a path in $(N, E)$ connecting node $i_{1}=r$ with node $i_{k}=j$ then $\left(i_{h}, i_{h+1}\right) \in D^{r}$ for all $h=1, \ldots, k-1$. Clearly, $x_{i}^{r}=v(\{i\})$ when node $i$ has no successor, and each term $x_{j}^{r}$ on the right hand side of (3.4) has been determined since $\tau(j)<\tau(i)$ when $j \in \operatorname{des}(i) \backslash\{i\}$. We can readily see by induction that it holds that for all $i \in N$

$$
x_{i}^{r}=v(\operatorname{des}(i))-\sum_{j \in \operatorname{Suc}(i)} v(\operatorname{des}(j))
$$

and therefore

$$
x^{r}(\operatorname{des}(i))=v(\operatorname{des}(i)) .
$$

See Figure 1. At allocation $x^{r}$ an agent gets what he contributes when he joins the descendants of his successors in arborescence $\left(N, D^{r}\right)$. Therefore for each $r \in N$, the tree solution $x^{r}$ with respect to node $r$ is a marginal contribution vector for some permutation.

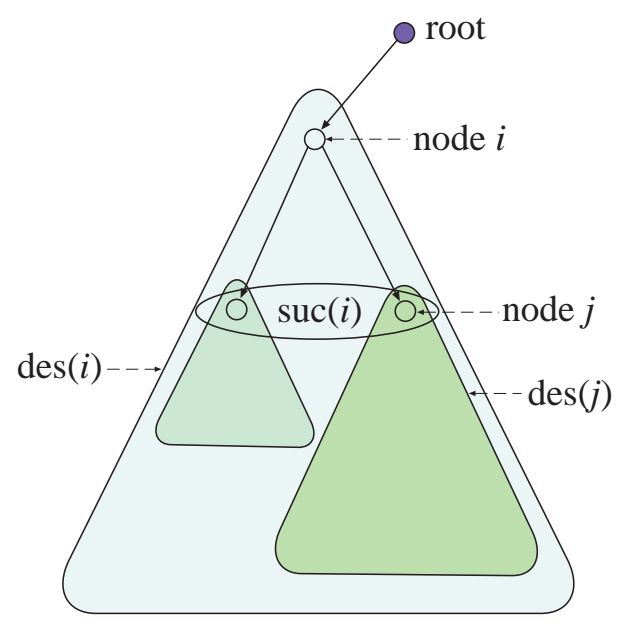

Figure 1: Tree solutions

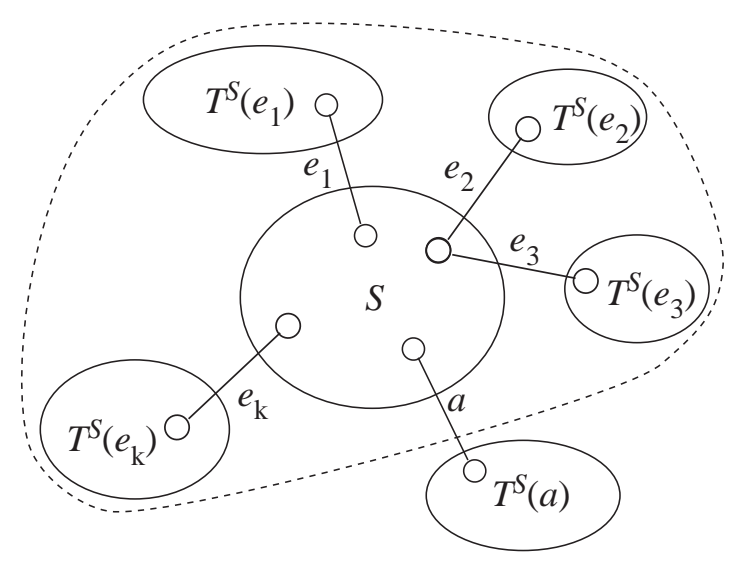

Figure 2: Connected node set

Definition 3.2. For an acyclic graph game $(N, v, E)$, the average tree solution, denoted $\operatorname{AT}(N, v, E)$, is the average of all tree solutions $x^{r}$ with respect to node $r \in N$, i.e.,

$$
\operatorname{AT}(N, v, E)=\frac{1}{n} \sum_{r \in N} x^{r} .
$$


It has been shown in Herings et al. [4] that the average tree solution can be axiomatized by efficiency and component fairness. The latter property says that if an edge is deleted the average loss for the two resulting components is the same, where the average is taken over all players in the component.

For a given acyclic connected undirected graph $(N, E)$, let $\delta(S), S \in C^{E}(N)$, be the set of edges in $E$ having one end node in $S$ and the other end node outside $S$, i.e.,

$$
\delta(S)=\{e \in E \mid e=\{i, j\}, i \in S, j \in N \backslash S\} .
$$

Deleting an edge $a$ of $\delta(S)$ results in two disjoint connected node sets, one containing the set $S$. We denote the component that does not contain the set $S$ by $T^{S}(a)$. We call $T^{S}(a)$ a satellite of $S$. See Figure 2. The set $S$ is connected to satellite $T^{S}(a)$ through the edge $a$. Notice that the union of $S$ and all its satellites equals the set of all nodes. The next theorem gives a sufficient condition such that the average tree solution is an element of the core.

Theorem 3.3. Suppose the acyclic graph game $(N, v, E)$ satisfies

$$
v(N) \geq v(S)+\sum_{e \in \delta(S)} v\left(T^{S}(e)\right) \quad \text { for all } S \in C^{E}(N)
$$

and

$$
v\left(N \backslash T^{S}(a)\right) \geq v(S)+\sum_{e \in \delta(S) \backslash\{a\}} v\left(T^{S}(e)\right) \text { for all } S \in C^{E}(N) \text { and } a \in \delta(S)
$$

Then the average tree solution $A T(N, v, E)$ is an element of the core, in particular the core $C(N, v, E)$ is nonempty.

Proof. We first prove that for every $r \in N$ the tree solution $x^{r}$ with respect to node $r$ is an element of $C(N, v, E)$. Take any node $r \in N$ and let $D^{r}$ and $x^{r}$ be the arborescence with root $r$ and the corresponding tree solution, respectively. Then we have $\operatorname{des}(r)=N$, which implies $x^{r}(N)=v(N)$ from (3.6). We will show that

$$
x^{r}(S) \geq v(S) \text { for all } S \in C^{E}(N) .
$$

Take any $S \in C^{E}(N)$. When the root $r$ is an element of $S$, by the construction of the tree solution, $x^{r}$ satisfies

$$
x^{r}\left(T^{S}(a)\right)=v\left(T^{S}(a)\right)
$$

for all $a \in \delta(S)$, see (3.5). By (3.7) the tree solution $x^{r}$ then satisfies

$$
x^{r}(S)=v(N)-\sum_{a \in \delta(S)} x^{r}\left(T^{S}(a)\right)=v(N)-\sum_{a \in \delta(S)} v\left(T^{S}(a)\right) \geq v(S) .
$$

When the root $r$ is not an element of $S, r$ is in $T^{S}(a)$ for some unique $a \in \delta(S)$. By construction

$$
x^{r}\left(N \backslash T^{S}(a)\right)=v\left(N \backslash T^{S}(a)\right)
$$

and for all $e \in \delta(S) \backslash\{a\}$ it holds that

$$
x^{r}\left(T^{S}(e)\right)=v\left(T^{S}(e)\right) .
$$


See for illustration the connected node sets circumscribed by a dotted circle in Figure 2. Therefore, by (3.8), we obtain

$$
\begin{aligned}
x^{r}(S) & =x^{r}\left(N \backslash T^{S}(a)\right)-\sum_{e \in \delta(S) \backslash\{a\}} x^{r}\left(T^{S}(e)\right) \\
& =v\left(N \backslash T^{S}(a)\right)-\sum_{e \in \delta(S) \backslash\{a\}} v\left(T^{S}(e)\right) \geq v(S) .
\end{aligned}
$$

Hence $x^{r} \in C(N, v, E)$ for every $r \in N$. Since $A T(N, v, E)$ is the average of all tree solutions, and the core is a convex set, the average tree solution $A T(N, v, E)$ is an element of the core.

Condition (3.7) states that the worth of the grand coalition should be at least equal to the worth of any connected coalition plus the sum of the worths of all its satellites. Condition (3.8) states that for any satellite of a connected coalition it holds that the worth of the players outside this satellite is at least equal to the worth of this coalition plus the sum of the worths of its other satellites.

Corollary 3.4. In an acyclic graph game $(N, v, E)$ satisfying conditions (3.7) and (3.8), for each node $r \in N$ the tree solution $x^{r}$ with respect to node $r$ is a marginal contribution vector, being an extreme point of the core.

Proof. The tree solution $x^{r}$ with respect to node $r \in N$ satisfies $x_{i}^{r}=v(\{i\})$ for node $i$ with $\tau(i)=0$ by (3.4). By the induction over the height $\tau(i)$ we see from this equality and (3.4) that $\sum_{j \in \operatorname{des}(i)} x_{j}^{r}=v(\operatorname{des}(i))$ holds for every node $i$. Therefore $x^{r}$ satisfies $n$ linearly independent inequalities out of those defining the core by equality, meaning that $x^{r}$ is an extreme point of the core.

Take a node $r \in N$ as root. For $j \in N \backslash\{r\}$, let $a_{j}^{r}$ be the unique edge of $\delta(\{j\})$ such that $j$ 's satellite $T^{\{j\}}\left(a_{j}^{r}\right)$ contains node $r$. Then the tree solution $x^{r}=\left(x_{1}^{r}, \ldots, x_{n}^{r}\right)$ with respect to node $r$ can be explicitly written as follows,

$$
\begin{aligned}
& x_{r}^{r}=v(N)-\sum_{e \in \delta(\{r\})} v\left(T^{\{r\}}(e)\right), \\
& x_{j}^{r}=v\left(N \backslash T^{\{j\}}\left(a_{j}^{r}\right)\right)-\sum_{e \in \delta(\{j\}) \backslash\left\{a_{j}^{r}\right\}} v\left(T^{\{j\}}(e)\right) \text { for } j \neq r .
\end{aligned}
$$

Hence, for $j \in N$, the $j$ th component of the average tree solution of the graph game $(N, v, E)$ is equal to

$$
\begin{aligned}
A T_{j}(N, v, E) & =\frac{1}{n}\left(v(N)-\sum_{a \in \delta(\{j\})} v\left(T^{\{j\}}(a)\right)\right) \\
& +\sum_{a \in \delta(\{j\})} \frac{\left|T^{\{j\}}(a)\right|}{n}\left(v\left(N \backslash T^{\{j\}}(a)\right)-\sum_{e \in \delta(\{j\}) \backslash\{a\}} v\left(T^{\{j\}}(e)\right)\right) .
\end{aligned}
$$

The first term between brackets reflects how much node $j$ contributes when he is joining all his satellites together, while the second term between brackets describes how much he contributes for linking a node in one of his satellites to the other satellites. The number 
$\left|T^{\{j\}}(a)\right|$ is the number of players in the satellite that is connected to node $j$ through link $a \in \delta(\{j\})$.

Herings et al. [4] show that for the class of super-additive acyclic graph games the average tree solution is always an element of the core. The next lemma shows that super-additivity implies conditions (3.7) and (3.8).

Lemma 3.5. A super-additive acyclic graph game $(N, v, E)$ satisfies both conditions (3.7) and (3.8).

Proof. Take any $S \in C^{E}(N)$ and let $\delta(S)=\left\{a_{1}, \ldots, a_{k}\right\}$. Clearly, for each $h=1, \ldots, k$, it holds that $S \cup T^{S}\left(a_{h}\right) \in C^{E}(N)$. Therefore, letting $T_{h}=\bigcup_{i=1}^{h} T^{S}\left(a_{i}\right)$, it holds that $S \cup T_{h} \in C^{E}(N)$ for $h=0,1, \ldots, k$, where we use the convention that $T_{0}=\emptyset$. From super-additivity it follows that for $h=1, \ldots, k$

$$
v\left(S \cup T_{h-1}\right)+v\left(T^{S}\left(a_{h}\right)\right) \leq v\left(S \cup T_{h}\right) .
$$

From this it follows that for all $h=1, \ldots, k$

$$
v\left(S \cup T_{h-1}\right)+\sum_{i=h}^{k} v\left(T^{S}\left(a_{i}\right)\right) \leq v\left(S \cup T_{h}\right)+\sum_{i=h+1}^{k} v\left(T^{S}\left(a_{i}\right)\right) .
$$

Since $S \cup T_{k}=N$, this implies

$$
v(S)+\sum_{i=1}^{k} v\left(T^{S}\left(a_{i}\right)\right) \leq \cdots \leq v\left(S \cup T_{k}\right)=v(N),
$$

from which (3.7) follows.

To prove condition (3.8), take any $a \in \delta(S)$ and let the edges $a_{1}, \ldots, a_{k}$ in $\delta(S)$ be indexed such that $a_{k}=a$. Then $S \cup T_{k-1}=S \cup\left(\cup_{h=1}^{k-1} T^{S}\left(a_{h}\right)\right)=N \backslash T^{S}(a)$. From (3.12) it follows that

$$
v(S)+\sum_{h=1}^{k} v\left(T^{S}\left(a_{h}\right)\right) \leq v\left(N \backslash T^{S}(a)\right)+v\left(T^{S}(a)\right),
$$

which implies (3.8).

Note that the conditions (3.7) and (3.8) do not impose any lower bound condition on $v(T)$ if the set $N \backslash T$ is not a satellite of any connected set. This fact is the reason that conditions (3.7) and (3.8) are weaker than the condition of super-additivity. For example, consider the case of four players and take $E=\{\{1,2\},\{2,3\},\{3,4\}\}$, then super-additivity requires that it must hold that $v(\{2\})+v(\{3\}) \leq v(\{2,3\})$. Since $\{1,4\}$ is not a satellite of $\{2,3\}$, this condition is not present in (3.7) or (3.8). If $E=\{\{1,2\},\{1,3\},\{1,4\}\}$, i.e., a star graph, then super-additivity requires $v(\{1\})+v(\{j\}) \leq v(\{1, j\})$ for all $j \neq 1$. Since the set $N \backslash\{1, j\}$ is not a satellite for any $j \neq 1$, these conditions are not present in (3.7) or (3.8), either.

Although all tree solutions are extreme points of the core, it is not the case that the core is always equal to the convex hull of all tree solutions. For example, if the game is convex, i.e., $v(S)+v(T) \leq v(S \cup T)+v(S \cap T)$, then all $n$ ! marginal contribution vectors are extreme points of the core and the number of different marginal contribution vectors is typically much larger than the number of different tree solutions, which is at most $n$. 


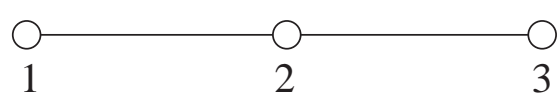

Figure 3: Communication structure

\section{Subcore}

In this section we introduce a refinement of the core of an acyclic graph game. From conditions (3.7) and (3.8) we see that when an acyclic graph game $(N, v, E)$ satisfies these conditions, then for every $S \in C^{E}(N)$ it holds that

$$
v(S) \leq \min \left\{\begin{array}{l}
v(N)-\sum_{a \in \delta(S)} v\left(T^{S}(a)\right) \\
\min _{a \in \delta(S)}\left\{v\left(N \backslash T^{S}(a)\right)-\sum_{e \in \delta(S) \backslash\{a\}} v\left(T^{S}(e)\right)\right\}
\end{array}\right\} .
$$

This motivates us to refine the core of an acyclic graph game as follows. Let us denote the right hand side of (4.1) by $w(S)$ with the convention that $w(N)=v(N)$.

Definition 4.1. For an acyclic graph game $(N, v, E)$, the subcore $S C(N, v, E)$ is given by

$$
S C(N, v, E)=\left\{\begin{array}{l|l}
x \in \mathbb{R}^{n} & \begin{array}{l}
x(N)=v(N) \\
x(S) \geq w(S) \text { for all } S \in C^{E}(N)
\end{array}
\end{array}\right\} .
$$

By definition it holds that the subcore is a subset of the core. More precisely, a payoff vector is an element of the subcore if the grand coalition receives its worth $(x(N)=v(N)$, efficiency) and for every other connected coalition $S$ it holds that (i) $S$ receives at least what it can get on its own $(x(S) \geq v(S)$, core), (ii) $S$ receives at least what it contributes when it joins its satellites to form the grand coalition $\left(x(S) \geq v(N)-\sum_{a \in \delta(S)} v\left(T^{S}(a)\right)\right.$ ), and (iii) $S$ receives at least what it contributes to the other satellites before a satellite of $S$ joins to form the grand coalition $\left(x(S) \geq v\left(N \backslash T^{S}(a)\right)-\sum_{e \in \delta(S) \backslash\{a\}} v\left(T^{S}(e)\right)\right.$, for all $\left.a \in \delta(S)\right)$. The idea is that the satellites of a connected set $S$ of players need $S$ to form the grand coalition, so that $S$ can claim a payoff at least equal to what it then contributes. The lowest of these contributions $(w(S))$ is the least what coalition $S$ wants to receive. The next theorem states that all such claims can be honored in the sense that the subcore of an acyclic graph game satisfying conditions (3.7) and (3.8) is nonempty because it always contains the average tree solution.

Theorem 4.2. For the class of acyclic graph games satisfying conditions (3.7) and (3.8) it holds that the average tree solution is an element of the subcore.

The theorem follows immediately from (4.1). Therefore on the class of acyclic graph games satisfying conditions (3.7) and (3.8) the subcore is a nonempty refinement of the core. Moreover, the tree solution with respect to any node, which is an extreme point of the core, remains an element of the subcore, and hence is an extreme point of the subcore.

The next example is a graph game with player set $N=\{1,2,3\}$ having limited communication structure represented by the graph in Figure 3. The characteristic function values are given by

$$
\begin{aligned}
& v(\{1\})=v(\{2\})=v(\{3\})=0 \\
& v(\{1,2\})=a ; v(\{2,3\})=b \\
& v(\{1,2,3\})=1,
\end{aligned}
$$




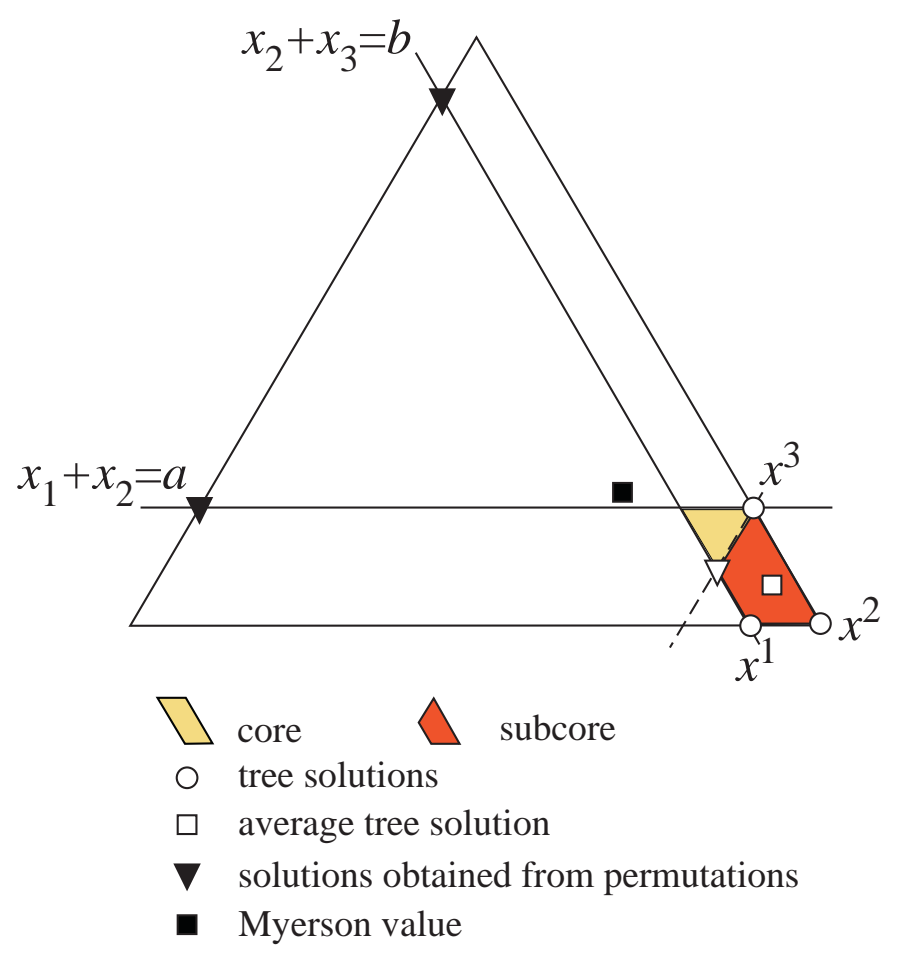

Figure 4: Core and tree solutions

with $0<a<b<1$. The core of this game is given by the linear system

$$
\begin{aligned}
& x_{1}, x_{2}, x_{3} \geq 0 \\
& x_{1}+x_{2} \geq a ; x_{2}+x_{3} \geq b \\
& x_{1}+x_{2}+x_{3}=1
\end{aligned}
$$

and is shown in Figure 4 for $a=0.8$ and $b=0.9$. The three tree solutions $x^{r}, r \in N$, are

Table 1: Tree solutions

\begin{tabular}{c|ccc} 
root $r$ & 1 & 2 & 3 \\
\hline$x_{1}^{r}$ & $1-b$ & 0 & 0 \\
$x_{2}^{r}$ & $b$ & 1 & $a$ \\
$x_{3}^{r}$ & 0 & 0 & $1-a$
\end{tabular}

given in Table 1 and are depicted in the figure by white circles. The average tree solution is therefore $((1-b) / 3,(1+a+b) / 3,(1-a) / 3)$, which is shown by the white square in the figure. The two black triangles show the marginal contribution vectors obtained for the permutations $(2,1,3)$ and $(2,3,1)$. Both vectors are not elements of the core. The black square in the figure denotes the Myerson value. The Myerson value is also outside the core. Notice that the second player who is pivotal in the communication graph gets more at the average tree solution than at the Myerson value. The dotted line is the additional constraint of the subcore, which has a new extreme point, indicated by the white triangle.

\section{Acknowledgment}

This research started while the second author was visiting the Department of Econometrics and Tinbergen Institute, Free University, Amsterdam, and the Department of Econometrics 
\& Operations Research and CentER, Tilburg University, Tilburg, the Netherlands under grant B 46-551 from the Netherlands Organization for Scientific Research (NWO). He is very grateful to the organization as well as both universities. He is also partially supported by JSPS Grant-in-Aid (B) 18310101, 2006.

\section{References}

[1] P. Borm, G. Owen, and S. Tijs: On the position value for communication situations. SIAM Journal of Discrete Mathematics, 5 (1992), 305-320.

[2] G. Demange: On group stability in hierarchies and networks. Journal of Political Economy, 112 (2004), 754-778.

[3] D.B. Gillies: Some Theorems on n-Person Games (Princeton University Press, Princeton, NJ, 1953).

[4] P.J.J. Herings, G. van der Laan, and D. Talman: The average tree solution for cycle-free graph games. Games and Economic Behavior, 62 (2008), 77-92.

[5] R.B. Myerson: Graphs and cooperation in games, Mathematics of Operations Research, 2 (1977), 225-229.

[6] L. Shapley: A value for n-person games. In H.W. Kuhn and A.W. Tucker (eds.): Contributions to the Theory of Games II, (Princeton University Press, Princeton, NJ, 1953), $307-317$.

[7] M. Slikker: A characterization of the position value. International Journal of Game Theory, 33 (2005), 505-514.

Yoshitsugu Yamamoto

Graduate School of Systems and Information Engineering University of Tsukuba

Tsukuba, Ibaraki 305-8573, Japan

E-mail: yamamoto@sk.tsukuba.ac.jp 